Urologe 2011 · 50:852

DOI 10.1007/s00120-011-2638-z

Online publiziert: 24. Juni 2011

(c) Springer-Verlag 2011

\author{
H. Rexer · S. Krege ${ }^{2}$ \\ ${ }^{1}$ AUO, Schwarz \\ ${ }^{2}$ Krankenhaus Maria Hilf, Krefeld
}

\title{
3. Europäisches Konsensusmeeting zum Hodentumor
}

\section{Vom 10.-12. November 2011 findet dieses Meeting in Berlin statt. Wir la- den Sie herzlich ein, diese Gelegen- heit zu nutzen und daran teilzuneh- men.}

Die Entwicklung der Therapie beim Hodentumor ist beispielhaft. Nach der Einführung der Cisplatin-basierten Polychemotherapie Mitte der 1970er Jahre als erstem großem Durchbruch waren es die Interdisziplinarität und die konsequente Durchführung von Studien, denen die hohen Heilungsraten beim Hodentumor anzurechnen sind. In Deutschland formierte sich bereits 1988 eine interdisziplinäre Gruppe, bestehend aus Urologen, internistischen Onkologen und Strahlentherapeuten sowie Pathologen. 1996 fand ein erstes Konsensusmeeting in Halle statt. Nach Erweiterung der Gruppe um Mitglieder aus den Organgruppen der AUO, AIO und ARO als Arbeitsgemeinschaften der Deutschen Krebsgesellschaft wurde die Hodentumorgruppe als erste interdisziplinäre Organgruppe der DKG etabliert.

Auf dem Krebskongress 2000 stellte die Gruppe den ersten evidenzbasierten Konsensus zur Diagnostik und Therapie beim Hodentumor vor. Da bereits zu diesem Zeitpunkt internationale $\mathrm{Zu}$ sammenarbeiten in Form von Studienprojekten bestanden, war es das nächste Ziel, einen Europäischen Konsensus zu erreichen. Dieses Ziel wurde erreicht. 2003 fand das erste Europäische Konsensusmeeting in Essen statt. 2006 richteten die niederländischen Kollegen das zwei- te Meeting in Amsterdam aus. Von Mal zu Mal konnte durch die Integration neuer Studienergebnisse eine weitere Standardisierung der Diagnostik und stadienspezifischen Therapie erreicht werden.

Im Brennpunkt der aktuellen Bemühungen steht eine Verbesserung der Behandlungsergebnisse von Rezidivtumoren und refraktären Tumoren. Dazu wurde ein Netzwerk auf nationaler und internationaler Ebene geschaffen. Parallel zu klinischen Studien arbeiten grundlagenforschungsorientierte Gruppen daran, Resistenzmechanismen aufzudecken. Somit wird beim Hodentumor die Forderung nach translationaler Forschung umgesetzt. Ein weiterer aktueller Schwerpunkt der Gruppe ist die Nachsorge beim Hodentumor. Diesbezüglich wurde gerade in den Fachzeitschriften der verschiedenen Fachdisziplinen ein in Kooperation mit den Schweizer Kollegen überarbeitetes Konzept vorgestellt, bei dem die Häufigkeit der Bildgebung deutlich vermindert wurde. Hierzu fehlt es jedoch noch weitgehend an Evidenz. Studien zur Nachsorge sind schwierig durchzuführen.

All dies wird Thema des 3. Europäischen Hodentumorkonsensus vom 10.-12. November 2011 in Berlin sein. Über 60 europäische Experten, Uroonkologen, internistische Onkologen, Radioonkologen und Pathologen werden die bereits im Vorfeld erarbeiteten konsensfähigen Empfehlungen zur Diagnostik und Therapie beim Hodentumor vorstellen sowie primär nicht konsensusfähige Themen und ungelöste Probleme im Plenum dis- kutieren. Als besonderes Highlight ist zudem der Gastvortrag von Larry Einhorn, dem Vater der PEB-Polychemotherapie, hervorzuheben.

Wer also die weitere Entwicklung beim Hodentumor hautnah erleben möchte, ist herzlich eingeladen, sich unter http:// www.european-consensus-conference.de über Details zu informieren und sich zu registrieren. Die Veranstaltung findet zudem in angenehmer Arbeitsatmosphäre im traditionsreichen Harnack-Haus im Grunewald statt. Und wer zwischendurch eine Auszeit braucht, wird hierzu sicherlich das richtige Angebot im pulsierenden Berlin finden.

\section{Korrespondenzadresse \\ H. Rexer \\ AUO, \\ Seestraße 11, \\ 17252 Schwarz \\ AUO@MeckEvidence.de}

Interessenkonflikt. Der korrespondierende Autor gibt an, dass kein Interessenkonflikt besteht.

Prof. Dr. S. Krege ist 2. Sprecherin der AUO. 